\title{
FACTORES RELACIONADOS CON LA DEPRESIÓN EN PACIENTES PEDIÁTRICOS CON CÁNCER Y PARTICIPACIÓN DE LA ENFERMERA EN SU DETECCIÓN
}

\section{FACTORS ASSOCIATED WITH DEPRESSION IN PEDIATRIC CANCER PATIENTS, AND PARTICIPATION OF NURSING IN ITS DETECTION}

\author{
TITULO CORTO: FACTORES RELACIONADOS CON LA DEPRESIÓN EN PACIENTES \\ PEDIÁTRICOS CON CÁNCER
}

Sandra Velásquez-Silva ${ }^{1}$ y Lina Zuluaga-Sarmiento ${ }^{2}$

Recibido en febrero 23 de 2015

Aceptado en mayo 11 de 2015

\section{RESUMEN}

El objetivo de este trabajo fue describir cuáles son los factores relacionados con la depresión en los pacientes pediátricos con cáncer y de qué manera la enfermería puede participar en su detección. Se realizó una revisión integrativa de artículos publicados entre 2000 y 2012. Se seleccionaron 15, se analizaron críticamente y organizaron por temas de acuerdo a su propósito. Los factores relacionados con la depresión que se encontraron son: personales: capacidad de adaptación, nivel de desarrollo y funcionamiento físico, nivel cognitivo y emocional, género, forma de afrontamiento, autoestima, apariencia e imagen corporal; familiares y sociales: apoyo familiar y apoyo social; y relacionados con la enfermedad y tratamiento: estancia hospitalaria, procedimientos médicos y de enfermería, aislamiento, etapa y efectos secundarios de la quimioterapia, calidad de la atención y la no identificación a tiempo de trastornos psicológicos. Se concluye que existen factores personales, familiares, de la enfermedad y tratamiento predictores y moduladores de la depresión que se relacionan con el riesgo o aparición de esta en el niño con cáncer. Dentro de las acciones de enfermería se destacan la valoración de los factores, la participación en grupos interdisciplinarios y la promoción de redes de apoyo social.

Palabras clave: depresión; pediatría; enfermería oncológica (Fuente:DeCS)

\section{Abstract}

The objective of this work is to describe what are the factors associated with depression in pediatric patients with cancer and how nurses can participate in its detection. We conducted an integrative review of articles published

\footnotetext{
1. Enfermera Especialista en Enfermería Oncológica. Enfermera Instituto Nacional de Cancerología. Bogotá-Colombia. Correo electrónico: svelasquez0528@hotmail.com

2. Enfermera Especialista en Enfermería Oncológica. Enfermera Instituto Nacional de Cancerología. Bogotá-Colombia. Correo electrónico: publicacion.enf.INC@gmail.com
} 
between 2000 and 2012. Fifteen articles were selected and then critically analyzed and organized by subjects according to their purpose. Among factors associated with depression are the following: personal factors: adaptability, developmental level and physical functioning, cognitive and emotional level, gender, form of coping and psychological reactions, self-esteem, appearance and body image and changes in lifestyle; family and social factors: family support and social support; factors related to the disease and treatment: hospitalization, medical and nursing procedures, insulation, stage of cancer disease, side effects of chemotherapy, quality of care and non-opportune identification of psychological disorders. We concluded that there are personal and family and disease and treatment factors that are constituted as predictors and modulators of depression and are related to the risk or on set of depression in pediatric patients with cancer. Within nursing actions include the assessment of the factors, participation in interdisciplinary groups and promoting social support networks.

Keywords: depression; pediatrics; nursing oncology (Fuente:DeCS)

\section{INTRODUCCIÓN}

$E_{s}^{1}$ cáncer pediátrico representa una problemática en salud mundial, registrándose 160.000 nuevos casos y 90.000 muertes anuales por esta causa ${ }^{1}$. En Estados Unidos, el cáncer es la principal causa de muerte por enfermedad en niños entre 0 y 15 años, con una tasa de incidencia de 15.8 X100.000, una tasa de mortalidad de $2.2 \mathrm{X} 100.000^{2}$ y una supervivencia relativa a 5 años del $83.1 \%$ en $2009^{3}$. En contraste, en países en vías de desarrollo como Colombia, las tasas de mortalidad por cáncer pediátrico son altas y las tasas de supervivencia relativa a cinco años son bajas ${ }^{4}$, aportando el $90 \%$ de los casos de muerte por cáncer pediátrico en el mundo 5 . En Colombia, la mortalidad por cáncer infantil ha disminuido en los últimos 20 años del 16,2\% al 8,3\%, sin embargo tal reducción aun no es comparable con los países desarrollados ${ }^{6}$.

Como respuesta a la problemática actual del cáncer pediátrico, se ha generado el Programa de Lucha Contra el Cáncer por la Organización Mundial de la Salud $(\mathrm{OMS})^{7}$, la inclusión del módulo de detección del cáncer en la estrategia Atención Integrada a la Enfermedades Prevalentes en la Infancia (AIEPI) de la OPS $^{8}$ y en Colombia, el Congreso de la República ha formulado la Ley 1388 del 26 de mayo de 2010, denominada "Por el Derecho a la Vida de los Niños con Cáncer en Colombia", además del Plan Decenal para el Control del Cáncer en Colombia 2012-2021 ${ }^{10}$ del Ministerio de la Protección Social y el Instituto Nacional de Cancerología. La respuesta mundial y local ha establecido como metas el diagnóstico temprano, el tratamiento, la rehabilitación, la paliación y la movilización social en torno al cáncer pediátrico, dentro de lo cual se contemplan los aspectos psicosociales asociados al cáncer pediátrico.

El cáncer pediátrico afecta a quien lo padece no solo a nivel físico, sino que puede acarrear enfermedades como la depresión ${ }^{11,12}$. La depresión influye en los pensamientos, sentimientos y la habilidad para funcionar en la vida diaria, que tiene factores de riesgo como la pérdida de la salud y en específico la amenaza de daño a la propia estructura física, psicológica y social del paciente pediátrico con cáncer ${ }^{13}$. La depresión afecta entre el 15\% y el $25 \%$ de los pacientes a quienes se les diagnostica el cáncer y a sus familiares, siendo más frecuente en los adolescentes que en los niños ${ }^{14}$.

Ante la depresión en el paciente pediátrico con cáncer, la enfermería debe dar una respuesta que desde la perspectiva del cuidado, para que pueda dar respuesta a las necesidades de esta población y de esta manera contribuir de forma efectiva a la mejora de su calidad de vida ${ }^{15,16}$.

A partir de la experiencia práctica en el servicio de pediatría del Instituto Nacional de Cancerología E.S.E., se ha observado que el paciente oncológico pediátrico es un individuo vulnerable a la depresión, la cual se genera por condiciones propias de la enfermedad, pero también por factores propios de su contexto y desarrollo, lo que en ocasiones influye en el éxito de su tratamiento.

De acuerdo con lo anterior, es de relevancia para la enfermería como disciplina profesional y objetivo de este estudio describir cuáles son los factores relacionados con la depresión en los pacientes pediátricos con cáncer 
y de qué manera la enfermería puede participar en su detección.

\section{MATERIALES Y MÉTODOS}

Se realizó una búsqueda de artículos de investigación publicados entre 2000 y 2012, en revistas indexadas en las bases de datos Scielo, PubMed, ScientDirect, Medline, Dialnet, Directory of Open Access Journal, Ovid MD, Nursing \& Allied Health Sourve (ProQuest), con el uso de los siguientes taxones: Niños, Adolescentes, Depresión, Enfermería, Soporte Social, Cáncer, Problemas psicosociales, Cuidado de Enfermería y sus descriptores equivalentes en inglés. Se seleccionaron artículos publicados en español e inglés.

La búsqueda arrojó un total de 1375 artículos de los cuales fueron pertinentes al tema 54. Las autoras dieron lectura por separado a los resúmenes de los artículos pertinentes y luego en conjunto llegaron a un consenso sobre la elegibilidad de las publicaciones.

Fueron seleccionados 15 artículos que cumplieron con los siguientes criterios: artículos que abordaran la depresión en pacientes pediátricos oncológicos y/o la participación de la enfermería en su detección, y artículos de investigación publicados en revistas indexadas entre los años 2000 y 2012.

Los 15 artículos seleccionados se organizaron en fichas de crítica de literatura científica de acuerdo con los criterios de $_{\text {Astete }}{ }^{17}$, las que permitieron evaluar la importancia, utilidad y novedad de los estudios, así como la coherencia interna de los mismos, el rigor de aplicación del método, el conocimiento generado y el cumplimiento de los principios éticos para la investigación con seres humanos.

Este estudio documental revisó de forma rigurosa que los estudios incluidos cumplieran con las pautas éticas internacionales para la investigación biomédica en seres humanos (CIOMS) y se rigió por principios éticos relacionados con el respeto por la propiedad intelectual.

\section{RESULTADOS}

Los hallazgos se agruparon en dos temas principales: factores relacionados con la depresión en pacientes pediátricos, y participación de la enfermería ante la detección de los factores. En la tabla 1 se describen los estudios identificados.

Tabla 1: Estudios identificados factores relacionados con la depresión

\begin{tabular}{|l|l|l|}
\hline \multicolumn{1}{|c|}{ ESTUDIO } & \multicolumn{1}{|c|}{ METODO } & \multicolumn{1}{c|}{ RESULTADOS } \\
\hline Hedstrom et al. ${ }^{18}$, & Cualitativo-Análisis de contenido & $\begin{array}{l}\text { El cáncer en la adolescencia se relaciona con } \\
\text { experiencias negativas como el miedo, el } \\
\text { temor a la muerte y alteraciones físicas. Las } \\
\text { experiencias positivas incluyen el buen trato } \\
\text { del equipo de salud. }\end{array}$ \\
\hline Ishibashi ${ }^{19}$, & $\begin{array}{l}\text { Los jóvenes presentan alta incertidumbre } \\
\text { sobre el cáncer, tienen más conflictos con } \\
\text { sus padres, son difíciles las discusiones sobre } \\
\text { planes a futuro o sobre la muerte. }\end{array}$ \\
\hline Corey et al. ${ }^{20}$, & $\begin{array}{l}\text { Estudio secundario basado en dos estudios documental de estudios } \\
\text { previos de diseño transversal. La muestra total } \\
\text { de los dos estudios fue de 199 participantes. } \\
\text { Se recolectó información cuantitativa con } \\
\text { instrumentos de síntomas y soporte social }\end{array}$ & $\begin{array}{l}\text { Existe una relación entre el soporte social por } \\
\text { los proveedores de salud y menores niveles } \\
\text { de insomnio. } \\
\text { El soporte social de familia, amigos y } \\
\text { proveedores de salud es un importante } \\
\text { predictor de la salud mental. } \\
\text { A mayor edad se evidencia más ansiedad y } \\
\text { perturbación del estado de ánimo. }\end{array}$ \\
\hline
\end{tabular}




\begin{tabular}{|c|c|c|}
\hline ESTUDIO & METODO & RESULTADOS \\
\hline Li et al. ${ }^{21}$ & $\begin{array}{l}\text { Cualitativo descriptivo. Se realizaron } \\
\text { entrevistas semiestructuradas a } 98 \text { niños de } \\
2 \text { unidades de oncología de Hong Kong }\end{array}$ & $\begin{array}{l}\text { Existe un elevado nivel de ansiedad inicial, } \\
\text { más de la mitad de los niños presentaron } \\
\text { síntomas depresivos durante la estancia } \\
\text { hospitalaria. Todos los niños expresan tristeza } \\
\text { y preocupación. }\end{array}$ \\
\hline Kiernan et al. ${ }^{22}$, & $\begin{array}{l}\text { Cualitativo-Fenomenológico. Se realizaron } \\
\text { entrevistas semiestructuradas a } 10 \text { médicos } \\
\text { y enfermeras }\end{array}$ & $\begin{array}{l}\text { El diagnóstico es devastador para las familias. } \\
\text { Temor y ansiedad son los sentimientos más } \\
\text { mencionados por los niños y sus familias. }\end{array}$ \\
\hline Chico et al..$^{23}$, & $\begin{array}{l}\text { Cualitativo-Análisis de contenido. Se } \\
\text { realizaron entrevistas semiestructuradas a } \\
10 \text { niños }\end{array}$ & $\begin{array}{l}\text { Los niños recuerdan la quimioterapia } \\
\text { principalmente por los efectos secundarios } \\
\text { que produce. A menudo se sienten solos y } \\
\text { aburridos. La hospitalización es vista como } \\
\text { una situación ansiogénica para el niño y su } \\
\text { familia. El hospital es visto de forma dual, por } \\
\text { un lado representa sufrimiento y por otro cura. }\end{array}$ \\
\hline Engvall et al. ${ }^{24}$, & $\begin{array}{l}\text { Cuantitativo descriptivo. Se incluyeron } 48 \\
\text { participantes }\end{array}$ & $\begin{array}{l}\text { El personal de salud sobreestima la utilización } \\
\text { de estrategias de afrontamiento de los } \\
\text { adolescentes a la enfermedad y el malestar } \\
\text { relacionado con el tratamiento. }\end{array}$ \\
\hline Cabrera et al. ${ }^{25}$, & $\begin{array}{l}\text { Cuantitativo correlacional. Se aplicaron } \\
\text { cuestionarios de ansiedad y depresión a } 65 \\
\text { participantes }\end{array}$ & $\begin{array}{l}\text { Se observó que existe una relación negativa } \\
\text { estadísticamente significativa entre la ansiedad } \\
\text { y el tiempo de diagnóstico. } \\
\text { No se encuentran diferencias entre los estados } \\
\text { emocionales de niños y niñas. }\end{array}$ \\
\hline Cavuşoğlu, ${ }^{26}$ & $\begin{array}{l}\text { Estudio cuantitativo descriptivo comparativo- } \\
100 \text { participante } 50 \text { con cáncer y } 50 \text { sanos }\end{array}$ & $\begin{array}{l}\text { Los niños con cáncer tienen mayores } \\
\text { puntuaciones de depresión que niños sanos }\end{array}$ \\
\hline Bragado et al. ${ }^{27}$, & $\begin{array}{l}\text { Cuantitativo descriptivo comparativo } 120 \\
\text { participantes } 30 \text { niños con cáncer y } 90 \text { niños } \\
\text { sanos sin historia de cáncer }\end{array}$ & $\begin{array}{l}\text { No existen diferencias entre las variables } \\
\text { estudiadas en los grupos. Los niños con } \\
\text { cáncer indican que gozan de menos salud y } \\
\text { flexibilidad que los niños sanos. La autoestima } \\
\text { predice la depresión, la salud y el auto } \\
\text { concepto predicen la ansiedad. }\end{array}$ \\
\hline Hernández et al. ${ }^{28}$, & $\begin{array}{l}\text { Cuantitativo descriptivo comparativo. } 50 \\
\text { participantes }\end{array}$ & $\begin{array}{l}\text { No existen diferencias significativas entre los } \\
\text { menores que se encuentran en tratamiento } \\
\text { activo y los que están fuera del tratamiento. } \\
\text { Los menores presentan problemas de ansiedad } \\
\text { seguidos por quejas somáticas y problemas } \\
\text { afectivos. En menor grado se presenta } \\
\text { problemas de conducta, déficit de atención e } \\
\text { hiperactividad. }\end{array}$ \\
\hline Matziou et al. ${ }^{29}$, & $\begin{array}{l}\text { Cuantitativo-descriptivo-comparativo. } 80 \\
\text { niños con cáncer y } 84 \text { niños sanos }\end{array}$ & $\begin{array}{l}\text { Aunque estudios anteriores muestran que } \\
\text { los niños con cáncer tienen alto riesgo de } \\
\text { depresión, este estudio encontró que no hay } \\
\text { diferencias en comparación con niños sanos. }\end{array}$ \\
\hline
\end{tabular}




\begin{tabular}{|c|c|c|}
\hline ESTUDIO & METODO & RESULTADOS \\
\hline Miller et al. ${ }^{30}$, & $\begin{array}{l}\text { Cuantitativo correlacional. } 75 \text { madres de niños } \\
\text { con cáncer }\end{array}$ & $\begin{array}{l}\text { El estrés se relacionó positivamente con la } \\
\text { depresión en el niño con cáncer. El afecto } \\
\text { negativo se asoció positivamente con la } \\
\text { ansiedad y depresión. El afrontamiento } \\
\text { moderó estas asociaciones. }\end{array}$ \\
\hline Kersun et al. ${ }^{31}$, & Cuantitativo descriptivo. 41 adolescentes & $\begin{array}{l}\text { Las tasas de auto reporte de ansiedad y } \\
\text { depresión son bajas, aunque los oncólogos } \\
\text { perciben más angustia en los pacientes. }\end{array}$ \\
\hline Rodríguez et al. ${ }^{14}$ & $\begin{array}{l}\text { Cuali-cuantitativo descriptivo. } 40 \text { adolescentes } \\
\text { valorados para determinar diagnósticos de } \\
\text { trastorno mental }\end{array}$ & $\begin{array}{l}65 \% \text { de los adolescentes valorados } \\
\text { presentaron un diagnóstico psiquiátrico, el } \\
42 \% \text { presentaron depresión. }\end{array}$ \\
\hline
\end{tabular}

\section{Factores relacionados con la depresión en pacientes pediátricos con cáncer}

En la revisión se encontraron 18 factores asociados con la depresión en pacientes pediátricos con cáncer. Estos factores se clasifican en personales, familiares y los relacionados con el tratamiento y la enfermedad.

\section{Factores personales}

En presencia de una enfermedad crónica y estresante como lo es el cáncer, hay factores que pueden significar unas alertas o alarmas para la identificación de depresión en niños y adolescentes. Existen factores personales que al ser correctamente identificados pueden alertar sobre la necesidad de establecer acciones para evitar consecuencias mayores en el futuro del tratamiento y en la vida del niño o adolescente. Dentro de estos factores se encuentran la adaptación, el nivel de desarrollo, el género, el afrontamiento, la autoestima y los cambios en el estilo de vida. A continuación se describe cada uno de ellos.

La capacidad de adaptación, pues una mala adaptación puede relacionarse con el riesgo de depresión ${ }^{23,27,28}$.

El nivel de desarrollo y funcionamiento físico y el nivel cognitivo y emocional, los cuales son aspectos que se relacionan con la edad; pues se da una expresión de sentimientos en función de la etapa del desarrollo ${ }^{23}$, encontrando que los adolescentes con cáncer presentan niveles más elevados de depresión que los niños ${ }^{25}$, 29 que evidencia que la corta edad puede ser un factor de protección ${ }^{26}$.
El género, pues hay estudios encuentran diferencias en los puntajes de depresión para hombres y mujeres; algunos afirman mayores niveles en los hombres ${ }^{14,25,26,29}$, mientras que otros no encuentran diferencias ${ }^{27}$.

La forma de afrontamiento y reacciones psicológicas (estrés), pues se evidenciaron asociaciones de estos con la depresión ${ }^{27,30}$.

La autoestima, pues esta se asocia con más salud y menor depresión ${ }^{27}$.

La apariencia e imagen corporal, pues esta genera un impacto y hace que se destaquen visualmente entre los pares $^{23,27}$.

Los cambios en los estilos de vida, pues esto genera gran impacto por el cambio en las rutinas ${ }^{23,25}$.

\section{Factores familiares y sociales}

A nivel familiar se destacan factores como el apoyo familiar y social. A continuación se describe cada uno de ellos.

Apoyo familiar: El apoyo de la familia es un elemento primordial dentro del tratamiento contra el cáncer de los niños y adolescentes, se vincula con el bienestar subjetivo y la salud mental. El apoyo familiar es visto como un factor protector ${ }^{14,23,25,27}$.

Apoyo social: El apoyo social inadecuado se asocia con la depresión ${ }^{26}$. 


\section{Factores relacionados con la enfermedad y el tratamiento}

Con relación a la enfermedad y el tratamiento se destacan la estancia hospitalaria, los procedimientos médicos y de enfermería, el aislamiento, la etapa de la enfermedad oncológica, los efectos secundarios de la quimioterapia, la calidad de la atención y la identificación de trastornos psicológicos. A continuación se describe cada uno de ellos.

La estancia (frecuencia de hospitalización, el tiempo de hospitalización), ya que el hospital es visto como un lugar no deseado, lo que produce ansiedad ${ }^{23}$, sentimientos de separación ${ }^{32}$ y estrés entendido como angustia ${ }^{18}$.

Los procedimientos médicos y de enfermería, pues producen dolor, ansiedad ${ }^{23,25}$ y estrés ${ }^{32}$. El aislamiento, ya que produce sentimientos de soledad ${ }^{23} \mathrm{y}$ se percibe como un cambio en la vida cotidiana ${ }^{25}$.

La etapa de la enfermedad oncológica (tiempo desde el diagnóstico), ya que se han evidenciado más reacciones psicológicas en las etapas iniciales, relacionadas con la crisis que se vive ante el diagnóstico, el inicio del tratamiento y aparición de efectos secundarios del mismo $^{28,29}$.

Los efectos secundarios de la quimioterapia, ya que estos se asocian con episodios depresivos en gran parte de los $\operatorname{niños}^{14,23,26,27}$.

La calidad de la atención prestada, pues la atención percibida como de calidad genera sentimientos positivos ${ }^{29}$.

La no identificación a tiempo de trastornos psicológicos o desequilibrio del estado mental, ya que esto aumenta el riesgo de depresión ${ }^{31}$.

\section{Participación de enfermería ante la detección de factores relacionados con la depresión en pacientes pediátricos con cáncer}

Teniendo en cuenta la problemática de la depresión en la población pediátrica con cáncer, surge la inquietud de buscar cuál es la participación de la enfermería para ayudar a detectar los factores de riesgo presentes en la depresión.

Se identificaron siete actividades realizadas por la enfermería a niños y adolescentes en tratamiento oncológico para detectar factores relacionados con depresión que son: permitir la expresión de sentimientos y emociones, participar en grupos interdisciplinarios

Valoración de la depresión a partir de técnicas e instrumentos, preparación mediante la educación frente al diagnóstico y tratamiento, juego terapéutico, promover el acceso a las redes de soporte familiar y social y mantener buenas relaciones con los niños y sus familias. A continuación se describen cada una de ellas.

Permitir la expresión de sentimientos y emociones: Esta actividad le da la oportunidad a la enfermería de reconocer las necesidades de los niños ${ }^{23}$, aunque algunos estudios ponen de manifiesto la falta de tiempo para llevar a cabo esta actividad ${ }^{30}$.

Participar en grupos interdisciplinarios: Esta actividad permite una atención integral al niño con cáncer ${ }^{14}$.

Valoración de la depresión a partir de técnicas e instrumentos: Esta actividad le da a la enfermera un dato objetivo del nivel de depresión o del riesgo de depresión que pueda presentar el niño $0^{25,29}$.

Preparación mediante la educación frente al diagnóstico y tratamiento: Esta actividad es un factor protector, pues da seguridad y control ${ }^{30}$, disminuye las consecuencias negativas ${ }^{14}$, contribuye a reducir el malestar físico ${ }^{31}$, brinda un escenario para la escucha de preocupaciones y temores ${ }^{29}$, permite a los niños convertirse en participantes activos de su cuidado, lo que les otorga un sentido de seguridad ${ }^{27}$ y la información suministrada reduce la incertidumbre y los sentimientos negativos ${ }^{26}$.

Juego terapéutico: Esta actividad provee un escenario propicio para suministrar información y para aumentar el desarrollo emocional y cognitivo ${ }^{31}$.

Promover el acceso a las redes de soporte familiar y social: Esta actividad promueve el continuar con la vida cotidiana previa al diagnóstico, favorece la interacción con familiares, allegados y pares, lo que les permite verbalizar sus sentimientos y preocupaciones ${ }^{28,31}$.

Mantener buenas relaciones con los niños y sus familias: Esta actividad favorece el desarrollo de confianza, empatía y la posibilidad de realizar preguntas y resolver dudas ${ }^{26,28}$.

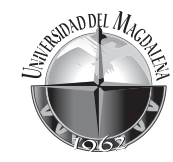




\section{DISCUSIÓN}

Existen factores personales que se asocian con la depresión, en este estudio, se destacó el género como uno de ellos, hecho que concuerda con Pascoe et al. ${ }^{32}$, quienes afirman que el ser mujer ha mostrado ser un factor predictor de la ansiedad y depresión; con Zelter et $a l .{ }^{33,} 34$ quienes destacan que dentro de los factores de riesgo en el estatus psicológico está el ser mujer y la presencia de una condición médica grave. Lo anterior puede deberse a que las mujeres han mostrado menor adaptación física y mental ${ }^{35}$.

Por su parte la autoestima, también se relaciona con la depresión, en este estudio como factor predictor, lo que contrasta con lo propuesto por Li et al. ${ }^{36} \mathrm{y} \mathrm{Li}$ et al. ${ }^{37}$ quienes lo destacan como un factor asociado que se da por el impacto del cáncer en la vida cotidiana de los niños. Von Essen et al. ${ }^{38}$, destacan que la depresión y la baja autoestima se presentan más en los niños que no reciben tratamiento y en los periodos posteriores al mismo, lo que sugiere la importancia del seguimiento continuo.

Factores de la enfermedad y el tratamiento se asocian con la propensión a la depresión; los niños con cáncer que reciben quimioterapia has mostrado estar más expuestos a la depresión que los niños sanos o que padecen otras enfermedades crónicas ${ }^{39}$, lo que es coherente con lo sucedido en los adultos ${ }^{40}$ y que contrasta con lo encontrado por Yallop et al. ${ }^{41}$, quienes encontraron que los niños con cáncer no eran más propensos que los niños sanos a tener una puntuación anormal en el bienestar psicosocial y en la subescala de depresión; sin embargo, O'Leary et al. ${ }^{42}$, ponen de manifiesto que los puntajes similares entre niños sanos y niños con cáncer pueden deberse a un sesgo en las respuestas, con tendencia a negar las dificultades.

La etapa de la enfermedad oncológica también es un factor que se asocia con la aparición de la depresión en el paciente con cáncer ${ }^{43}$, los niños con enfermedades crónicas y que requieren hospitalizaciones para su tratamiento son más propensos a la depresión que niños con enfermedades agudas ${ }^{44}$; así mismo, problemas como la ansiedad son más comunes en el momento del diagnóstico y la depresión suele ser más común luego del primer año ${ }^{45}$.

Frente a los efectos secundarios de la quimioterapia, el estudio de Vahdaninia et al. ${ }^{46}$, apoya el hecho de que síntomas como la fatiga es un factor de riesgo para el desarrollo de ansiedad y depresión; además este trabajo confirma la mayor prevalencia de depresión en estadíos iniciales del cáncer.

Dentro de las acciones a desarrollar con los niños con depresión, se destaca el apoyo social, más aún cuando los pacientes con cáncer manifiestan necesidades insatisfechas de apoyo social y baja utilización de los servicios psicosociales disponibles ${ }^{47}$, además de que el apoyo social y la depresión han mostrado correlacionarse de forma negativa, es decir que a mayor soporte social, menor depresión ${ }^{48}$. Programas de intervención en afrontamiento y resolución de problemas han mostrado ser efectivos para mejorar el estrés y los síntomas depresivos no solo en los niños sino en sus madres ${ }^{49}$. El apoyar a las familias es una intervención básica para la prevención e intervención en la depresión de los niños con cáncer, ya que se ha demostrado que los padres con ansiedad y depresión influyen en las respuestas de los niños ${ }^{50}$.

Una de las actividades angulares es la valoración de la depresión, dentro de ella se destaca la utilización de escalas e instrumentos sencillos, adaptados a la edad de los niños, válidos y confiables ${ }^{51}$.

Por su parte, el juego terapéutico ha demostrado ser eficaz en la identificación de habilidades de afrontamiento, la adaptación psicológica y la depresión. Dentro del juego terapéutico se incluye la educación frente al diagnóstico y tratamiento ${ }^{52}$.

\section{CONCLUSIONES}

Existen factores personales, familiares y de la enfermedad y tratamiento que se relacionan con el riesgo o aparición de depresión en el paciente pediátrico con cáncer. Dichos factores pueden constituirse en predictores o moduladores de la depresión.

Para la enfermería es de interés reconocer o detectar dichos factores, esto con el fin de mitigarlos o potenciarlos. Dentro de las acciones de enfermería que se destacan en este ámbito están el permitir la expresión de sentimientos y emociones, el participar en grupos interdisciplinarios, la valoración de la depresión a partir de técnicas e instrumentos, la preparación mediante la educación frente al diagnóstico y tratamiento, el juego terapéutico, 
el promover el acceso a las redes de soporte familiar y social y el mantener buenas relaciones con los niños y sus familias.

\section{AGRADECIMIENTOS}

Al Grupo de Investigación de Cuidado al Paciente Crónico y su Familia de la Facultad de Enfermería de la Universidad Nacional de Colombia, en el Marco del Programa para disminuir la carga de la enfermedad crónica en Colombia, por el acompañamiento en la estructuración de los elementos metodológicos para desarrollar este artículo.

\section{REFERENCIAS BIBLIOGRÁFICAS}

1. Parkin D, Bray F, Ferlay J, Pisani P. Global cancer statistics. CA Cancer J Clin. 2005 Mar-Apr; 55(2):74-108.

2. National Cancer Institute. Surveillance Epidemiology and End Results. Incidencia y Mortalidad por edad [internet]. [Consultado 2014 Jul 18]. Disponible en: http://seer.cancer.gov/csr/1975_2010/browse_csr. php? section $=28 \&$ page $=$ sect_28_table.01.html

3. National Cancer Institute. Surveillance Epidemioogy and End Results. Supervivencia relativa a 5 años. [internet]. [Consultado 2014 Jul 18]. Disponible en: http://seer.cancer.gov/csr/1975_2010/browse_csr. php? section $=28 \&$ page $=$ sect_28_table.08.html

4. Howard S, Metzger M, Wilimas J, Quintana Y, Pui C, Robison $\mathrm{L}$ et al. Childhood cancer epidemiology in low-income countries. Cancer. 2008 Feb; 112(3):461-72.

5. Sullivan R, Kowalczyk, Agarwal B, Ladenstein R, Fitzgerald E, Barr R et al. New policies to address the global burden of childhood cancers. The Lancet. 2013 Mar; 1 4(3):125-135.

6. Piñeros M, Gamboa O, Suarez A. Mortalidad por cáncer infantil en Colombia durante 1985 a 2008. Revista Panamericana de Salud pública. 2011 Jul; 30(1):15-21.

7. Organización Mundial de la Salud. Programa de la OMS de Lucha contra el Cáncer [internet]. [Consultado 2014 Jul 18]. Disponible en: http://www.who.int/cancer/es/

8. Organización Mundial de la Salud. El cáncer infantil en las Américas [internet]. [Consultado $2014 \mathrm{Jul}$ 18]. Disponible en: http://new.paho.org/hq/index. php?option $=$ com_docman\&task $=$ doc_details\&gid $=1$ $8352 \& \mathrm{mpl}=$ component\&itemid $=270 \& l a n g=$ en

9. Congreso de la República de Colombia. Ley 1388 del 26 de mayo de 2010 [internet]. [Consultado $2014 \mathrm{Jul}$ 18]. Disponible en: http://web.presidencia.gov.co/ leyes/2010/mayo/ley138826052010.pdf

10. Ministerio de Salud, Instituto Nacional de Cancerología E.S.E. Plan Decenal para el Control del Cáncer en Colombia 2012-2021 [internet]. [Consultado 2014 Jul 18].
Disponible en: http://www.cancer.gov.co/documentos/ Plandecenalparaelcontroldelcancer/PlanDecenal_ ControlCancer_2012-2021.pdf

11. Ruland C, Hamilton G, Schjoodt-Osmo B. The Complexity of Symptoms and Problems Experienced in Children with Cancer: A Review of the Literature. Journal of Pain and Symptom Management. 2009 Mar; 37(3): 403-418.

12. Dodd M, Janson S, Facione N, Faucett J, Froelicher ES, Humphreys J, et al. Advancing the science of symptom management. Journal of Advanced Nursing. $2001 \mathrm{Mar}$; 33(5):668- 676 .

13. González Y. Depresión en niños y niñas con cáncer. Actualidades en Psicología. 2006; 20(107): 22-44.

14. Rodríguez V, Sánchez C, Rojas N, Arteaga R. Prevalencia de trastornos mentales en adolescentes con cáncer. Revista Venezolana de Oncología. 2009 Dic; 21 (4): 29-34.

15. Lafaurie M, Caviedes M, Cortés C, Guzmán A, Hernández M, Rubio D. Historias de vida de niños con cáncer: construcción de significado y sentido. Revista Colombiana de Enfermería. 2007; 2(2): 55-65.

16. Vanegas B, Beltrán M, Cifuentes V, Duarte Y, Montoya $\mathrm{J}$, Rivera J, et al. Vivencia psicosociales reveladas por niños que reciben tratamiento con quimioterapia por cáncer. Avances en Enfermería. 2009; 27(2): 102-12.

17. Astete B. Lectura crítica de artículos originales en salud. Medicina de Familia. 2001; 2 (1). [internet]. [Consultado 2015 Feb 28]. Disponible en: http://www.samfyc.es/ Revista/v2n1/081-090.pdf

18. Hedström M, Skolin I, von Essen L. Distressing and positive experiences and important aspects of care for adolescents treated for cancer. Eur J Oncol Nurs. 2004 Mar; 8(1):6-17.

19. Ishibashi A. The needs of children and adolescents with cancer for information and social support. CancerNurs. $2001 \mathrm{Feb} ; 24(1): 61-7$.

20. Corey AL, Haase JE, Azzouz F, Monahan PO. Social support and symptom distress in adolescents/ young adults with cancer. J Pediatr Oncol Nurs. 2008 Sep-Oct; 25(5):275-84.

21. Li HC, Chung OK, Chiu SY. The impact of cancer on children's physical, emotional, and psychosocial wellbeing. Cancer Nurs. 2010 Jan-Feb; 33(1):47-54.

22. Kiernan G, Meyler E, Guerin S. Psychosocial issues and care in pediatric oncology: medical and nursing professionals' perceptions. Cancer Nurs. 2010 Sep-Oct; 33(5):12-20.

23. Chico E, Castanheira L, García R. Niños y adolescentes con cáncer: experiencias con la quimioterapia. Rev Latino-Am Enfermagem. 2010 Sep-Oct; 18(5).

24. Engvall G, Skolin I, Mattsson E, Hedström M, von Essen L. Are nurses and physicians able to assess which strategies adolescents recently diagnosed with cancer use to cope with disease- and treatment-related distress? Support Care Cancer. 2011 May; 19(5): 605-11. 
25. Cabrera P, Urrutia B, Vera V, Alvarado M, Vera P. Ansiedad y depresión en niños diagnosticados con cáncer. Revista de Psicopatología y Psicología Clínica. 2005; 10(2): 115-124.

26. Cavuşoğlu H. Depression in children with cancer. J Pediatr Nurs. 2001; 16(5):380-5.

27. Bragado C, Hernandez M, Sánchez M, Urbano S. Autoconcepto físico, ansiedad, depresión y autoestima en niños con cáncer y sanos sin historia de cáncer. Psicothema. 2008; 20(3): 413-419.

28. Hernández S, López C, Durá E. Indicadores De Alteraciones Emocionales Y Conductuales En Menores Oncológicos. Psicooncología. 2009; 6(2-3): 311-325.

29. Matziou V, Perdikaris P, Galanis P, Dousis E, Tzoumakas K. Evaluating depression in a sample of children and adolescents with cancer in Greece. Int Nurs Rev. 2008 Sep; 55(3): 314-9.

30. Miller KS, Vannatta K, Compas BE, Vasey M, McGoron KD, Salley CG, Gerhardt CA. The role of coping and temperament in the adjustment of children with cancer. J Pediatr Psychol. 2009 Nov-Dec; 34(10): 1135-43.

31. Kersun LS, Rourke MT, Mickley M, Kazak AE. Screening for depression and anxiety in adolescent cancer patients. J Pediatr Hematol Oncol. 2009 Nov; 31(11):835-9.

32. Pascoe S, Edelman S, Kidman A. Prevalence of psychological distress and use of support services bycancer patients at Sydney hospitals. Aust N Z J Psychiatry. 2000 Oct; 34(5): 785-91.

33. Zeltzer LK, Lu Q, Leisenring W, Tsao JC, Recklitis C, Armstrong G, et al . Psychosocial outcomes and healthrelated quality of life in adult childhoodcancer survivors: a report from the childhood cancer survivor study. Cancer Epidemiol Biomarkers Prev. 2008; 17 (2): 435-46.

34. Zeltzer LK1, Recklitis C, Buchbinder D, Zebrack B, Casillas J, Tsao JC, et al. Psychological status in childhood cancer survivors: a report from theChildhood Cancer Survivor Study. J Clin Oncol. 2009: 10; 27(14): 2396-404.

35. Chan CW, Choi KC, Chien WT, Cheng KK, Goggins W, So WK, et al. Health-related qualityof-life and psychological distress of young adultsurvivors of childhood cancer in Hong Kong. Psychooncology. 2014; 23(2): 229-36.

36. Li HC, Chung OK, Ho KY, Chiu SY, Lopez V. A descriptive study of the psychosocial well-being and quality of life ofchildhood cancer survivors in Hong Kong. Cancer Nurs. 2012; 35(6): 447-55.

37. Li HC, Lopez V, Joyce Chung OK, Ho KY, Chiu SY. The impact of cancer on the physical, psychological and social wellbeingof childhood cancer survivors. Eur J Oncol Nurs. 2013; 17(2): 214-9.

38. Von Essen L, Enskär K, Kreuger A, Larsson B, Sjödén PO. Self-esteem, depression and anxiety among Swedish children and adolescents on and off cancer treatment. Acta Paediatr. 2000; 89 (2): 229-36.

39. Arabiat DH, Elliott B, Draper P. The prevalence of depression in pediatric oncology patients undergoing chemotherapy treatment in Jordan. J Pediatr Oncol Nurs. 2012; 29(5): 283-8.

40. Yang YL, Liu L, Wang Y, Wu H, Yang XS, Wang JN, Wang L. The prevalence of depression and anxiety among Chinese adults withcancer: a systematic review and meta-analysis. BMC Cancer. 2013: 22; 13: 393.

41. Yallop K, McDowell H, Koziol-McLain J, Reed PW. Self-reported psychosocial wellbeing of adolescent childhood cancersurvivors. Eur J Oncol Nurs. 2013; 17(6): 711-9.

42. O'Leary TE, Diller L, Recklitis CJ. The effects of response bias on self-reported quality of life amongchildhood cancer survivors. Qual Life Res. 2007; 16(7): 1211-20.

43. Mhaidat NM, Alzoubi KH, Al-Sweedan S, Alhusein BA. Prevalence of depression among cancer patients in Jordan: a national survey. Support Care Cancer. 2009; 17(11): 1403-7.

44. Esmaeeli MR, Erfani Sayar R, Saghebi A, Elmi S, Rahmani S, Elmi S, Rabbani Javadi A. Screening for depression in hospitalized pediatric patients. Iran J Child Neurol. 2014; 8(1): 47-51.

45. Myers RM, Balsamo L, Lu X, Devidas M, Hunger SP, Carroll WL et al. A prospective study of anxiety, depression, and behavioral changes in the first year after a diagnosis of childhood acute lymphoblastic leukemia: a report from the Children's Oncology Group. Cancer. 2014; 120(9): 1417-25.

46. Vahdaninia M, Omidvari S, Montazeri A. What do predict anxiety and depression in breast cancer patients? A follow-up study. Soc Psychiatry Psychiatr Epidemiol. 2010; 45(3): 355-61.

47. Ernstmann N, Neumann M, Ommen O, Galushko M, Wirtz M, Voltz R, Hallek M, Pfaff H. Determinants and implications of cancer patients' psychosocial needs. Support Care Cancer. 2009; 17(11): 1417-23.

48. Bayat M, Erdem E, Gül Kuzucu E. Depression, anxiety, hopelessness, and social support levels of the parents of children with cancer. J Pediatr Oncol Nurs. 2008; 25(5): 247-53.

49. Melnyk BM, Alpert-Gillis L, Feinstein NF, Crean HF, Johnson J, Fairbanks E, et al . Creating opportunities for parent empowerment: program effects on the mental health/coping outcomes of critically ill young children and their mothers. Pediatrics. 2004; 113(6): e597-607.

50. Harper FW, Peterson $\mathrm{AM}^{2}$, Albrecht TL $L^{1}$, Taub JW'3 , Phipps $S^{4}$, Penner LA ${ }^{1}$. Posttraumatic Stress Symptoms in Parents of Pediatric Cancer Patients: A Mediational Analysis. J Trauma Stress Disord Treat. 2014; 3(4). [internet]. [Consultado 2015 Feb 28]. Disponible en: http://www. ncbi.nlm.nih.gov/pmc/articles/PMC4286796/ 
51. Liu Y, Wang J, Hinds PS, Wang J, Shen N, Zhao $X$, Ding $J$, Yuan $C$. The emotional distress of children with cancer in China: an item response analysis of C-Ped-PROMIS Anxiety and Depression short forms. [internet]. [Consultado $2015 \mathrm{Feb} 28$ ]. Disponible en: http://download.springer.com/static/pdf/538/ art\%253A10.1007\%252Fs11136-014-0870-x.pdf?auth6
$6=1425576494 \_3 \mathrm{~b} 4272 \mathrm{~b} 5758052 \mathrm{a} 7 \mathrm{f0d} 99 \mathrm{e} 5284561 \mathrm{a} 33$ \&ext $=. p d f$

52. Wiener L, Battles H, Mamalian C, Zadeh S. ShopTalk: a pilot study of the feasibility and utility of a therapeutic boardgame for youth living with cancer. Support Care Cancer. 2011; 19(7): 1049-54.

Para citar este artículo: Velásquez-Silva S, Zuluaga-Sarmiento L. Factores relacionados con la depresión en pacientes pediátricos con cáncer y participación de la enfermera en su detección. Duazary. 2015 dic; 12 (2): 164 - 173 